\title{
Uncertainties Modeling and Simulation of an Emergency Process with Fuzzy Petri Nets
}

\author{
Patrick Lallement \\ Charles Delaunay Institute, UMR CNRS n 6281 , University of Technology of Troyes, Troyes, France
}

\section{Email address:}

patrick.lallement@utt.fr

\section{To cite this article:}

Patrick Lallement. Uncertainties Modeling and Simulation of an Emergency Process with Fuzzy Petri Nets. International Journal of Management and Fuzzy Systems. Vol. 3, No. 1, 2017, pp. 1-9. doi: 10.11648/j.ijmfs.20170301.11

Received: May 4, 2016; Accepted: February 24, 2017; Published: March 11, 2017

\begin{abstract}
This paper describes a way to model and simulate an emergency procedure with uncertainties. These uncertainties (especially due to visibility conditions, stress of actors) may have a strong influence on operational decisions and lead to a bad efficiency of the emergency system, due to a wrong resources management. These variables are considered and processed as fuzzy numbers and they are used as input of a simulation model with fuzzy Petri nets to evaluate the reactivity and the efficiency of the procedure.
\end{abstract}

Keywords: Organizational Modelling, Uncertainties, Petri Nets, Fuzzy Simulation

\section{Introduction}

Emergency systems require tools and metrics to evaluate their procedures [1-4]. The performance term could be inappropriate when the goal is to save lives with a minimum of means [5]. Performance indicators in crisis situations revolve around following factors: flexibility, responsiveness and efficiency. Nevertheless, it is necessary to define that in the context of a crisis or a major accident is meant by these terms. The efficiency is not here relative on customer satisfaction, the term "beneficiary" would be better, i.e. someone who is in danger. Flexibility is the ability to adapt to unforeseen circumstances. Reactivity was stronger than in a business situation character, because delays can cause casualties. Combining flexibility and responsiveness means adaptability. More criteria can be used [6], [7]: agility that combines responsiveness, flexibility, efficiency (as defined in logistics [8]). Relevance results of the confrontation between means and objectives. The most relevant indicator for an emergency team in a crisis situation is the reactivity, which corresponds to the response time plus the evacuation time. The aim is to adapt the response mode and resources, taking into account that the inventory is not a priori known.

This paper considers a context of decision support for medical aid in case of emergency following a serious accident or a disaster. It is more of a help to the event management as an aid to crisis management. For emergency medical service, the subject is not to manage the crisis but to respond to a serious event, and in this case the threshold is considered at level 2 (at least one death [9]). In an emergency situation, standard procedures are applied, unlike in crisis where there is not necessarily a predefined procedure, or if there is one, perhaps it is no more applicable and actors will have to improvise as the situation is difficult to predict [10]. The standard procedure considered is a quarterly exercise in which you play a scenario. Actors therefore apply a procedure and test the predicted response to a major accident according to the procedure defined but by placing it in an uncertain environment: intervention conditions (weather, traffic, communication) and the state of mind of actors i.e. the limited capacity to understand and control events [11] and the stress [12], [13]. Individual behaviors lead then to organizational consequences because of biased appraisals [14], [15] and because of the short time for decision making [16].

The main goal of this paper is to represent these uncertainties as input of a simulation model of the procedure so that a simulation could aid for decision-making, especially for human resources management. In a context of emergency system, the decision will concern logistics in general and more particularly resources management based on situation and needs estimations. In the first part the emergency procedure is modeled, the uncertain variables are identified with their interrelationships. The second part describes a Petri-net-based model of the procedure and how the 
uncertainty of input variables is represented, the conditions of fuzzy simulation. The third part presents how the output variables can be obtained from a fuzzy simulation and how to interpret the results. Methods and results are then discussed for future works.

\section{The Emergency Process}

\subsection{The Procedure}

The medical emergency team called SAMU (Service Emergency Medical Assistance) applies a generic procedure model, which is used during exercises. It is composed of the following entities:

a) A rear-base $(\mathrm{RB})$ : coordination, decision-making

b) A communication center (CC): it centralizes information and exchanges with the RB.

c) The first team (FT): first intervention, it sends the first situation report to the $\mathrm{CC}$.

d) The advanced medical post (AMP), which deals with worst cases (medical care, evacuation) and sends the final report to the $\mathrm{CC}$.

The emergency exercise procedure can be decomposed in specific tasks:

$\mathrm{T} 1) \mathrm{CC}$ receives an alert from a source $\mathrm{S}$, the source can be one of the actors involved in the exercise,

T2) FT is sent to the scene of an emergency first aid

T3) FT sends a first impression report.

T4) FT enforcement, depending on the first perception of the situation

T5) The first report is sent to the $\mathrm{CC}$,

T6) AMP emergency, medical care and evacuation of the worst cases,

T7) Sending the full report on the situation to the CC.

\subsection{Uncertainty Factors}

Context parameters will affect the overall response time. A fairly general inventory is presented Table 1 . These factors may have a strong impact on particular steps of the procedure, as it is given Table 2. If the communication resources do not work, it will delay the transmission of reports. Environmental factors and accessibility factors may increase the access time (transport). Environmental, visibility, human and material resources factors may lengthen the duration of the in situ intervention. These factors are of different nature:

a) Quantitative with operational effects, which lead to additional delays: failure, limited resources, difficult traffic, limited bandwidth to communicate.

b) Quantitative due to biased decisions: unfavorable weather conditions and limited visibility can lead to underestimate a situation and in this case the needs may be bad-evaluated, which leads to waste time and resources.

c) Qualitative with a cause-and-effect relationship in terms of time and efficiency. This is the case of stress. It can overestimate the seriousness of the situation and also lose time in the intervention. In addition, stress may depend on other factors, unforeseen accidents, traffic constraints, limited visibility, number of victims, severity and limited experience.

\subsection{Procedure Modelling}

The advantage of modeling is to enable the control center (CC) to provide the influences he has, to make evolve the course of the procedure from reports that are returned in order to estimate the resources to be committed (FT and AMP), to fulfill an efficiency objective based on the need (number of victims). We can separate first three types of evaluation, which can be grouped under the terms of: responsiveness, accessibility and efficiency. These groups are each subject to different influences:

E1) Reactivity: it can be estimated by the communication and information processing delays, alerting, FT reporting, complete reporting if necessary.

E2) Accessibility: it will concern the delay between a decision to intervene and the arrival on site.

E3) Efficiency: it will concern in situ operations, medical care and report elaboration.

Table 1. Reactivity factors.

\begin{tabular}{ll}
\hline Factor class & Factors \\
\hline Availability & breakdown, accident \\
Accessibility & roads conditions, flood, landslide \\
Material resources & vehicles, logistics \\
Environment & weather conditions \\
Human resources & stress, skill, experience \\
Communication & network availability \\
Visibility & between actors, actors and environment \\
\hline
\end{tabular}

Table 2. Factors vs. procedure steps.

\begin{tabular}{ll}
\hline Factor class & Procedure steps \\
\hline Availability & $\mathrm{T} 2, \mathrm{~T} 4, \mathrm{~T} 6$ \\
Accessibility & $\mathrm{T} 2, \mathrm{~T} 4, \mathrm{~T} 6$ \\
Material resources & $\mathrm{T} 2, \mathrm{~T} 4, \mathrm{~T} 6$ \\
Environment & $\mathrm{T} 2, \mathrm{~T} 4, \mathrm{~T} 6$ \\
Human resources & $\mathrm{T} 4, \mathrm{~T} 6$ \\
Communication & $\mathrm{T} 1, \mathrm{~T} 2, \mathrm{~T} 3$ \\
Visibility & $\mathrm{T} 2, \mathrm{~T} 4, \mathrm{~T} 6$ \\
\hline
\end{tabular}

Influence factors which are concerned are:

F1) Responsiveness: communication delay (if unavailable access network), decision delay.

F2) Accessibility: state of roads, weather conditions, resources availability.

F3) Efficiency: visibility, resources capacity (quantitative but also qualitative).

Technical problems that hinder communication lead to a severe loss of time. Otherwise, responsiveness is a factor of organizational types. It can be considered that the responsiveness is worst in case of the first alert than after a post-report decision. Emergency teams are able to appreciate this reaction time. F2 factors relate to both random (breakdown, accident) and uncertain (roads, weather) factors. The state of the roads have their own influence factors: traffic and weather conditions. Fig. 1 gives an idea of the interrelationships between these factors.

The accessibility may be increased comparing to a G. P. S. (Global Positioning System) forecast. Some influence 
parameters can be known and have a predictable effects (urban density, time, state of the roads) but others will have unpredictable effects in terms of accessibility, especially when parameters cumulate their effects. Type 3 factors (F3) are rather qualitative. Fig. 2 shows out their interrelationships.

They are also heterogeneous. Skill, experience, stress can be expressed in qualitative (linguistic) terms but their resulting effect is very uncertain.

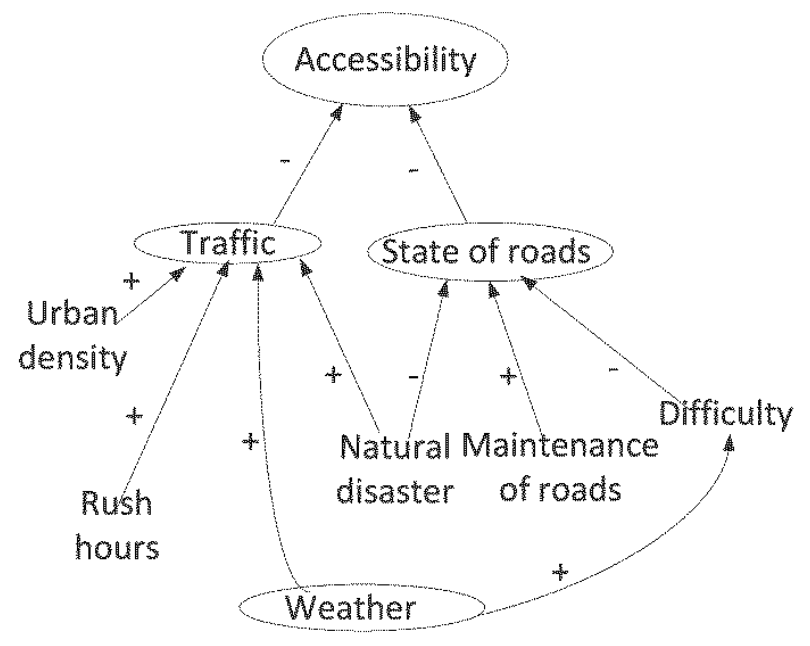

Figure 1. Accessibility-related parameters (+/- $\equiv$ decreases/increases).

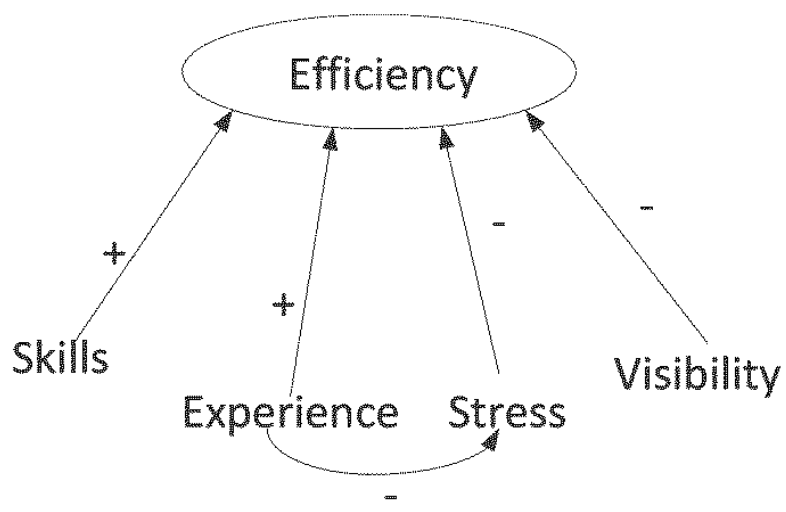

Figure 2. Efficiency related parameters.

\section{The Simulation Model}

\subsection{The Emergency Procedure as Discrete Event System}

The emergency procedure can be viewed as a discrete event system (DES). A DES is a dynamic system whose states changing will depend on the occurrence of events [17]. Discrete event systems can be modeled by a finite state automaton [18] or a Petri net, which offer the significant advantages of a graphical and a mathematical representation of DES.

The state graph of the emergency procedure can be made in Fig. 3. States represent resources levels (FT, FT + and PMA).

Depending on the report made by the FT team, an FT enforcement may be requested $(\mathrm{FT}+)$ before PMA, due to a bad-handled situation. State changes occur when:

a) decision are taken by the rear base RB depending on the reports of situation

b) emergency teams (first, first enforced, advanced medical task force) are operating.

The transition between FT and FT+, FT and PMA becomes uncertain. Discrete event systems can be crisp or fuzzy, depending on the conditions associated to the states. Recently an emergency procedure has been represented by a fuzzy finite state automaton to handle imprecise and uncertain data, and build a learning diagnosis [19] of the situation. Our purpose is quite different. It is not to model a crisis situation but a procedure and then, to be able to simulate different scenarios or strategies (for comparison). This problem is close to the performance issues in industrial systems, except that indicators are quite different for an emergency procedure as explained in introduction.

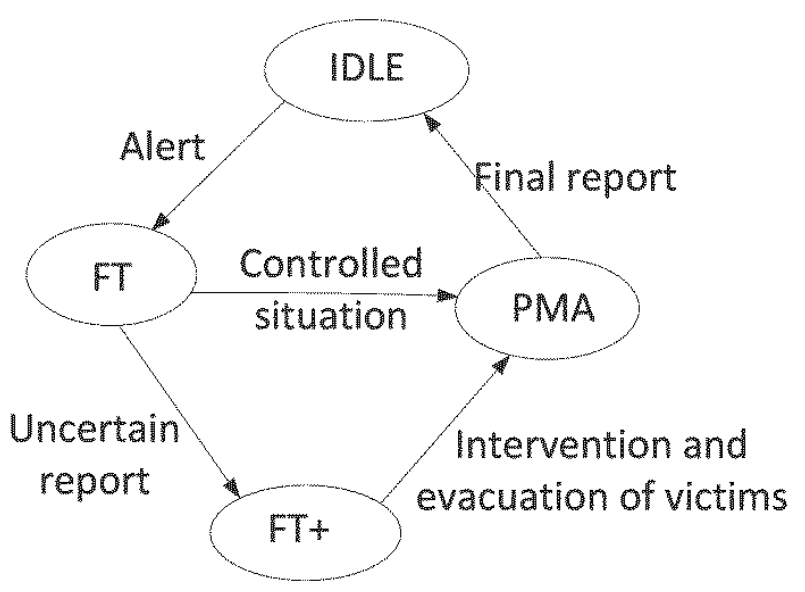

Figure 3. Automaton of the emergency procedure.

\subsection{The Emergency Procedure as Discrete Event System}

The graph states of the procedure can be represented by a colored Petri net $\mathrm{PN}_{1}$ given Fig. 4. Transitions $\mathrm{T}_{1}, \mathrm{~T}_{2}, \mathrm{~T}_{3}$ and $\mathrm{T}_{4}$ represent the different states changes. Specific criteria can also be attached to each of them: the post-alert responsiveness $\left(T_{1}\right)$, accessibility $\left(T_{2}\right)$ and in situ operations $\left(\mathrm{T}_{3}\right)$. The firing delay of these transitions may vary according to the influence parameters $\mathrm{F} 1$ (for $\mathrm{T}_{1}$ ), F2 (for $\mathrm{T}_{2}$ ) and F3 (for $\mathrm{T}_{3}$ ). Each team is represented by a specific color:

$\mathrm{c}_{1}$ : FT (first team)

$c_{2}$ : FT+ (first team enforced, according to the first report) $\mathrm{c}_{3}$ : AMP

The Petri net $\mathrm{PN}_{2}$ given Fig. 5 represents the medical care process of the victims. Three levels of seriousness are defined: the victims are not much $\left(\mathrm{g}_{1}\right)$, a few $\left(\mathrm{g}_{2}\right)$, severely $\left(\mathrm{g}_{3}\right)$ hit and each of them is represented by a corresponding color in $\mathrm{PN}_{2}$. The real configuration is initialized in $\mathrm{P}_{5}$ with $\left(\mathrm{N}_{1}, \mathrm{~N}_{2}, \mathrm{~N}_{3}\right)$, that is the number of victims corresponding to the $\left(\mathrm{g}_{1}, \mathrm{~g}_{2}, \mathrm{~g}_{3}\right)$ levels. Only FT and FT + teams can deal with $\mathrm{g}_{1}$ and $\mathrm{g}_{2}$. Only the AMP can deal with $\mathrm{g}_{3}$ level. $\mathrm{M}_{1}, \mathrm{M}_{2}$ and $\mathrm{M}_{3}$ are boolean messages, which inform about the operating team, which is deduced of the equivalent color marking in $\mathrm{P}_{3}$ (noted $\left.\left|\mathrm{P}_{3}\left(\mathrm{c}_{\mathrm{i}}\right)\right|, \mathrm{i}=1,2,3\right)$. When the marking of $\mathrm{P}_{5}$ with the 
colors $\mathrm{g}_{1}$ and $\mathrm{g}_{2}$ becomes null $\left(\left|\mathrm{P}_{5}\left(\mathrm{~g}_{1}\right)\right|=0\right.$ AND $\left.\left|\mathrm{P}_{5}\left(\mathrm{~g}_{2}\right)\right|=0\right)$, the FT and $\mathrm{FT}+$ teams become ineffective and the corresponding tokens $\mathrm{c}_{1}$ and $\mathrm{c}_{2}$ enter $\mathrm{P}_{4}\left(\mathrm{M}_{8}=\right.$ true $)$. When the marking of $\mathrm{P}_{5}$ is null $\left(\left|\mathrm{P}_{5}().\right|=0\right)$ the emergency process can be closed (final report, message $\mathrm{M}_{9}=$ true). The total intervention delay can be estimated from the arrival time of the last token in $\mathrm{P}_{5}$. The unit delays of treatments for $\mathrm{g}_{1}, \mathrm{~g}_{2}$ and $g_{3}$ respectively are represented by the firing delays of $T_{5}$, $\mathrm{T}_{6}$ and $\mathrm{T}_{7}\left(\mu_{1}, \mu_{2}\right.$ and $\mu_{3}$ respectively).

The Petri net $\mathrm{PN}_{3}$ given Fig. 6 represents more particularly the influence of the visibility and stress factors on the running operational process. Both factors will have an impact on the first report made by FT, which can lead to the decision of a FT reinforcement $(\mathrm{FT}+)$. The colors $\mathrm{s}_{0}$ and $\mathrm{v}_{0}$ are used when stress and visibility are normal, colors are set to $s_{1}$ and $\mathrm{v}_{1}$ in the contrary.

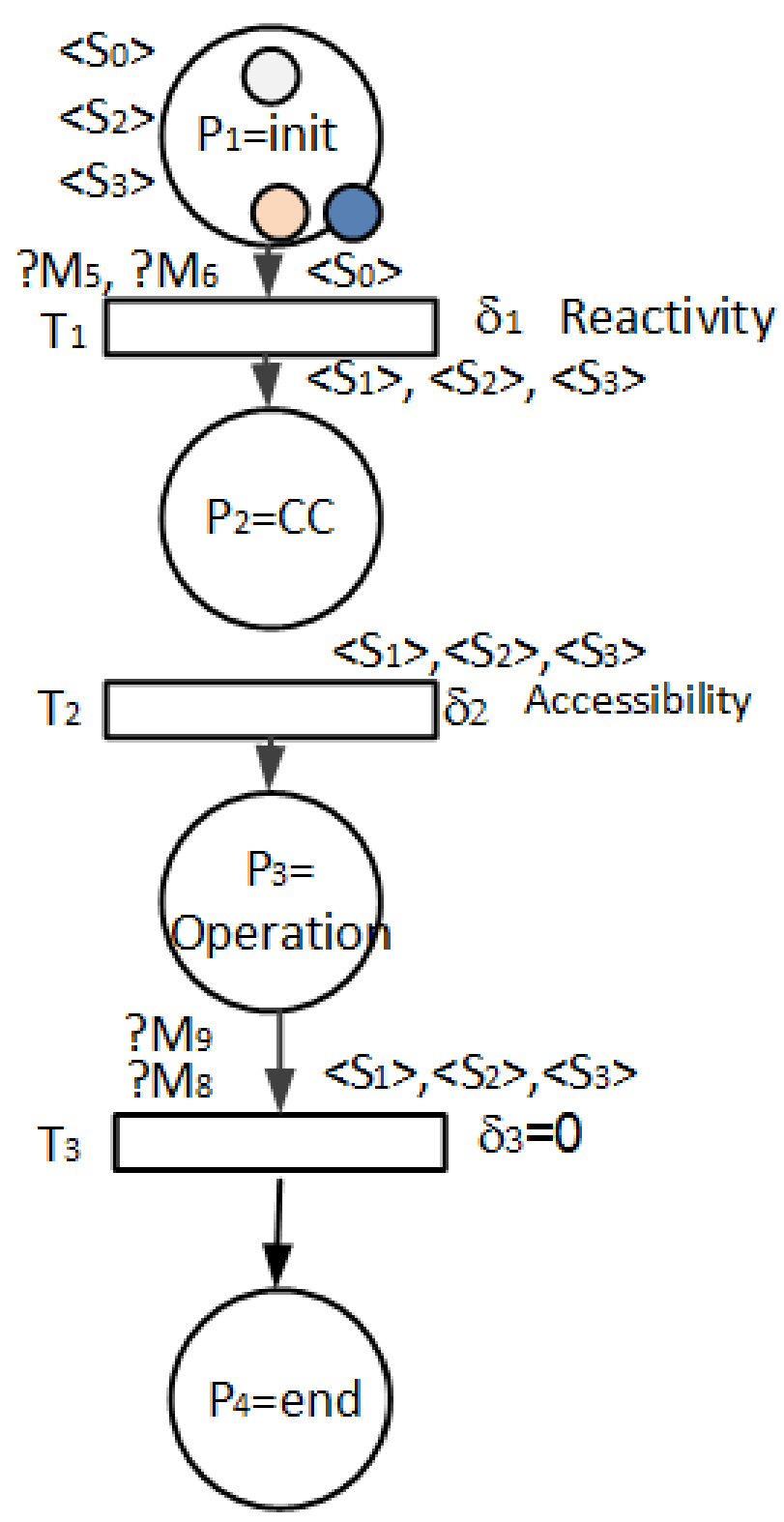

Figure 4. PN1 State graph of the procedure.

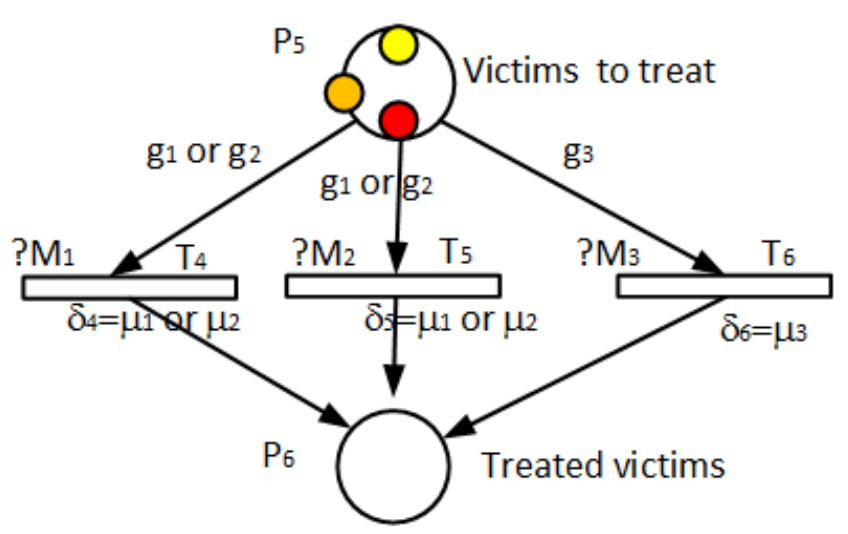

Figure 5. PN2 Medical care of victims.
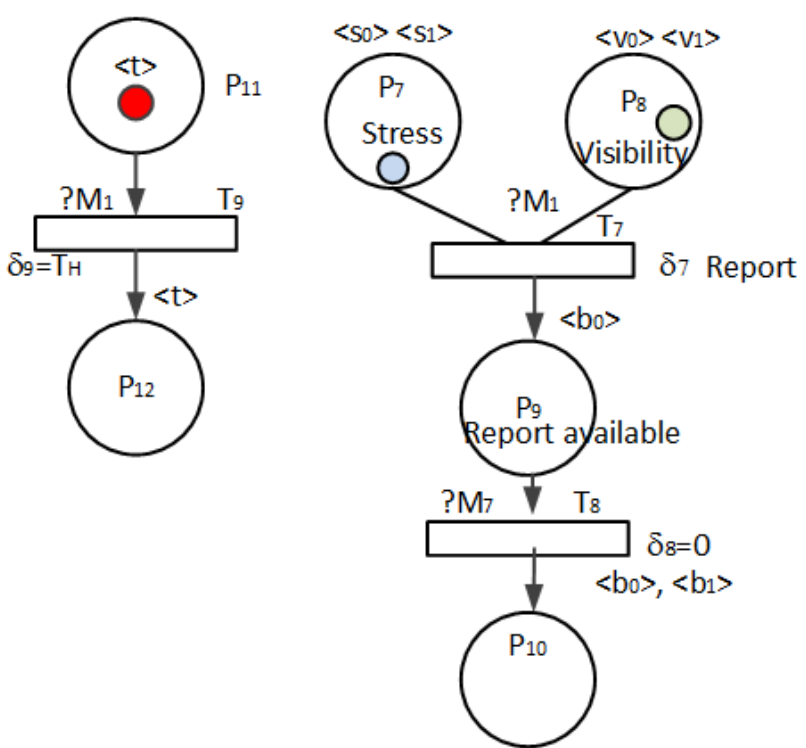

Figure 6. PN3, Report elaboration.

If the report elaboration delay ( $\mathrm{T}_{7}$ firing delay) exceeds a given threshold, this is considered as a negative report (token color $b_{1}$, which corresponds to a FT reinforcement (message $\mathrm{M}_{6}$ true, the token " $\mathrm{t}$ " reaches the place $\mathrm{P}_{12}$ ). When the report is positive the corresponding token color is $b_{0}$ and no FT+ team is requisite $\left(\mathrm{M}_{6}=\right.$ true $)$. All synchronization messages $\mathrm{M}_{\mathrm{i}}$ between $\mathrm{PN}_{1}, \mathrm{PN}_{2}$ and $\mathrm{PN}_{3}$ are summarized Table 3 .

\subsection{The Representation of Input Data Uncertainties}

The firing delays of transitions $\mathrm{T}_{1}, \mathrm{~T}_{2}$ and $\mathrm{T}_{7}, \delta_{1}, \delta_{2}$ and $\delta_{7}$ respectively, represent the reactivity, accessibility and evaluation/report delays. In particular, $\delta_{3}$ has a significant contribution in the total delay of the emergency procedure and in the emergency system efficiency. The first evaluation of the post-accident situation may be biased by the stress and/or by bad visibility conditions, so that a reinforcement of the first team may be requisite which is not relevant. In the contrary, bad visibility conditions can lead to underestimate a situation in terms of number and gravity of victims.

In an elementary approach, it is considered that the time spent to converge to a first report is significant of the bad conditions that the first team (FT) discovers in situ. It means 
that as this time increases, the report will tend to be negative and will request a reinforcement $(\mathrm{FT}+)$. This cause-and-effect relationship is represented in $\mathrm{PN}_{3}$ by a trigger threshold $(\mathrm{TH})$ and the firing delay of $\mathrm{T}_{9}$.

These delays and especially $\delta_{3}$ can be approximated with average values or [ $\mathrm{min}, \mathrm{max}]$ intervals but it seems not relevant to use ad hoc probability distributions because these delays are the cumulated result of many factors, some are of organizational or of human nature. For these reasons, it therefore seems interesting and relevant to represent them by fuzzy numbers.

Table 3. Synchronization messages.

\begin{tabular}{ll}
\hline Messages & Semantics \\
\hline M1 & $|\mathrm{P} 3(\mathrm{c} 1)|>0$ \\
M2 & $|\mathrm{P} 3(\mathrm{c} 2)|>0$ \\
M3 & $|\mathrm{P} 3(\mathrm{c} 3)|>0$ \\
M4 & $|\mathrm{P} 9()|>0$. \\
M5 & $|\mathrm{P} 10(\mathrm{~b} 0)|>0$ \\
M6 & $|\mathrm{P} 10(\mathrm{~b} 1)|>0$ \\
M7 & $|\mathrm{P} 12(\mathrm{t})|>0$ \\
M8 & $|\mathrm{P} 5(\mathrm{~g} 1)|=0$ AND $|\mathrm{P} 5(\mathrm{~g} 2)|=0$ \\
M9 & $|\mathrm{P} 5(\mathrm{~g} 3)|=0$ \\
\hline
\end{tabular}

The fuzzy subset theory [20] associated to the possibility theory [21] [22] allows to represent bad-known or uncertain data. The membership function, which characterizes a fuzzy data, presents a semantics of possibility or uncertainty [23]. A variable $X$ whose average value and confidence interval cannot be estimated from statistical tools can be represented with a membership function to a fuzzy subset, which represents here a subset of values from the considered universe $U$, which is the one of the real numbers. In this case, it is the subset of all possible values for $X$ that we want to measure, and it corresponds to the fuzzy assertion: " $X$ is approximately equal to $x$ ". The membership function can be viewed as a possibility measure to belong to this subset.

Thus the $\delta_{2}$ delay, which represents the logistic delay (arrival in site), can be represented by a fuzzy data as in Fig. 7, where the membership ad hoc (triangular) function $T(x, a$, b) represents the fuzzy assertion: "the access time is approximately equal to $x "$. One can assume that $x$ increases and the support $[a, b]$ becomes wider (the uncertainty increases) as the access conditions deteriorate (they may also cumulate their effects). As reference, normal conditions $C_{N}$ can be estimated with the estimated delay provided by a G. P. $\mathrm{S}$. Degradations sources come from weather conditions $\mathrm{C}_{\mathrm{w}}$ (storm, snow, ice), traffic conditions $\mathrm{C}_{\mathrm{t}}$ (urban density, hour), natural disaster $\mathrm{C}_{\mathrm{n}}$.

Data encapsulated in an triangular membership functions $\mu(x)=(x, a, b)$ can be obtained from experience of actors (emergency services are often requested, at any time) which is a potential source of data. Fuzzy conditions are cumulative, the resulting membership function (for ex. from elementary ones as in Fig. 7) can be obtained by classical operations of fuzzy variables aggregation:

$$
\mu(y)=\mu\left\{\cup_{\mathrm{i}}\left(C_{i}\right)\right\}=\max _{i}\left\{x_{i}\right\}
$$

\subsection{Fuzzy Petri Nets}

The combination between Petri nets and the subset theory has been proposed in the 90's with the general term of "fuzzy Petri nets". Despite the fact there are different approaches, two application classes have emerged:

a) the fuzzy control: Petri nets enable to represent a sequence of rules (by firing transitions [24]),

b) the observation and the characterization of a dynamic system, with uncertain and imprecise knowledge; in this case the marking represents the uncertain knowledge of the system state at a given time [25].

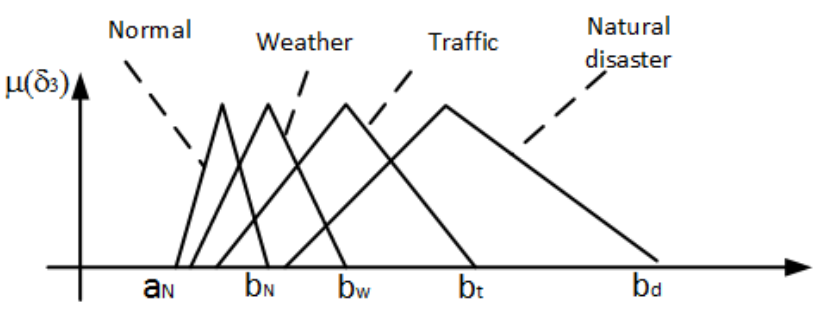

Figure 7. Fuzzy access delay representation.

Variables that capture this vagueness depend on the semantics associated to the Petri nets items: token, places. The fuzzy marking of a PN can be associated to a fuzzy number of token in a place. The fuzzy marking can be associated to the uncertain presence of a token in a place. A token may have a fuzzy location in a set of places where it is supposed to be (generally it is assumed that it can be in a given place and therefore, its location is associated with a possibility distribution).

When a place is associated to a logical proposition, the token present may have as attribute a membership function, which represents the truth degree of that proposition.

If we consider the marking changes after a transition has been fired, in these different approaches, two cases will result generally:

1) The token represents an entity and the transition firing represents a state changing, as it corresponds to a removal of tokens in the input places.

2) The firing represents a logical rule execution and after, the logical proposition remains valid, there is no removal of tokens in this case.

In our case, we represent a dynamic system and in its PN model, tokens represent entities, places represent a given step of a process, transitions represent states changing. Uncertainties affect some firing delays, which means that the input and output markings can be considered as fuzzy.

\subsection{Fuzzy Simulation}

A fuzzy simulation needs to generate values from a fuzzy subset. A convenient way is given by the "Fuzzy Numerical Simulation" or FNS method [26]. A first random draw is made according to a uniform law $U(0,1)$ to generate a number $t$, which will correspond to a $t$-cut. A second random number $y$ is generated in this interval according to a uniform 
law defined with:

$$
G_{t}=\left\{x, \mu_{\pi}(x) \geq t\right\}
$$

The fuzzy number $\pi$ is then replaced by this numerical value. This method is similar to the classical probabilistic approach. It presents the advantage of linearity [26]. Nevertheless, it amounts to work with an implicit probability distribution specified by the fuzzy subset. There are also other ways to approximate Monte Carlo simulation, by replacing the probability distributions by uncertainty functions, more general but handier to use. One combine then the interval calculus with the one of probabilities, to obtain an interval of the targeted probabilities [28].

In [29], the authors use an experimental plan to estimate the response surfaces of the simulation with fuzzy input data. Another way is used in this paper, already experienced in [30]. The first step is similar as to obtain an histogram by a Monte Carlo simulation. From a given number $N$ of the system's runs, the min and $\max$ values of a given variable $Y$ are calculated. The min-max interval is then decomposed in $n$ classes and for each of them, the frequency is calculated. This is the common way to estimate a probability distribution of $Y$. In a second step, a probability to possibility transform is used, to normalize the obtained distribution so that a possibility measure will correspond to an upper bound of probability. The algorithm used is given in [31].

Let $\left\{\mathrm{u}_{\mathrm{i}}\right\}$ the different classes used to approximate the probability distribution of $\mathrm{Y}$.

Let $\pi\left(\mathrm{u}_{\mathrm{i}}\right)=\pi_{\mathrm{i}}$ and $\mathrm{p}\left(\mathrm{u}_{\mathrm{i}}\right)$ their possibility and probability values respectively, so that:

$$
\mathrm{p}_{1}=0 \leq \ldots \leq \mathrm{p}_{\mathrm{n}} \leq \mathrm{p}_{\mathrm{n}+1}
$$

Then we calculate:

$$
\pi_{1} \leq \pi_{2} \leq \ldots \leq \pi_{\mathrm{n}} \leq \pi_{\mathrm{n}+1}=1
$$

The transform is used as follows:

$$
\pi_{\mathrm{i}}=\sum_{\mathrm{j}} \mathrm{p}_{\mathrm{j}}, \mathrm{j}=1, \ldots, \mathrm{n}
$$

Simulation and results

\subsection{Input Data}

Firing delays of $T_{1}, T_{2}$ and $T_{7}$ are represented by fuzzy numbers and their possibility distributions are given Fig. 8.

The trigger threshold has been tuned to $T_{H}=17.5$, which corresponds to the intersection between normal and stress situations. The unit delays of medical care is represented by uniform probability distributions $U(a, b)$, depending on the gravity (see table 4). They correspond to the treatment of one victim by a specific team (FT, FT+ and AMP).

\subsection{Evaluation of the Total Delay}

The tested configuration $C_{I}$ corresponds to $\left(N_{1}, N_{2}, N_{3}\right)=$ $(14,4,2)$. Fig. 9 shows the possibility distribution of the total response time $T$ (from alert to the end), in normal conditions (without degradations). Both "modes" can be explained as follows: each time FT + is requested $\left(\delta_{7}>T_{H}\right)$, the human resources capacity increases so that $g_{1}$ and $g_{2}$ victims are "served" faster. This corresponds to the fuzzy delay $(T \approx 261)$.

Table 4. Fuzzy time to treat one victim.

\begin{tabular}{lll}
\hline Gravity & Team & $\boldsymbol{U}(\boldsymbol{a}, \boldsymbol{b})$ \\
\hline g1 & FT, FT+, AMP & $\mathrm{U}(16,40)$ \\
g2 & FT, FT+, AMP & $\mathrm{U}(21,70)$ \\
g3 & AMP & $\mathrm{U}(51,100)$ \\
\hline
\end{tabular}
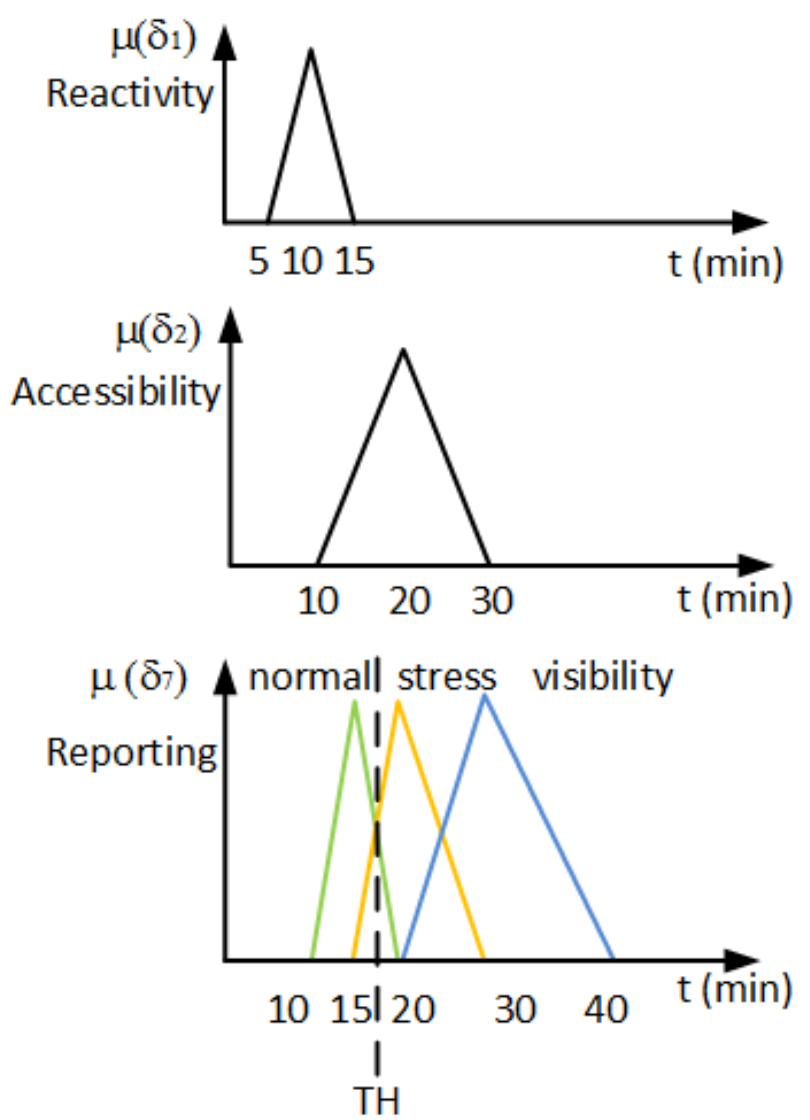

Figure 8. Fuzzy delay of $\delta 1, \delta 2$ and $\delta 7$.

In others cases, i.e. $\delta_{7} \leq T_{H}$, the total delay increases ( $T \approx 424)$. In case of more severe configurations, i.e. when $\mathrm{N}$ $\left(\mathrm{g}_{3}\right)$ increases then the result would increase severely because the AMP arrival time would be delayed. With bad visibility conditions, one can see Fig. 10 that for the same configuration $C_{l}$, the delay decreases because $\mathrm{FT}+$ is then systematically requested.

\subsection{Evaluation of the Procedure Efficiency}

In a first approach, we can estimate that the efficiency is maximum when all task forces (FT, FT+ and AMP) are always operating from their arrival time and the end of the procedure. As FT and $\mathrm{FT}+$ are affected to $\mathrm{g}_{1}$ and $\mathrm{g}_{2}$ victims, and AMP to $g_{3}$, it means that the efficiency decreases a) when FT and FT+ have finished while AMP is continuing or b) when AMP has finished when FT/FT+ is still operating. An 
efficiency measure can be directly obtained from the $\mathrm{P}_{3}$ marking (see Fig. 11).

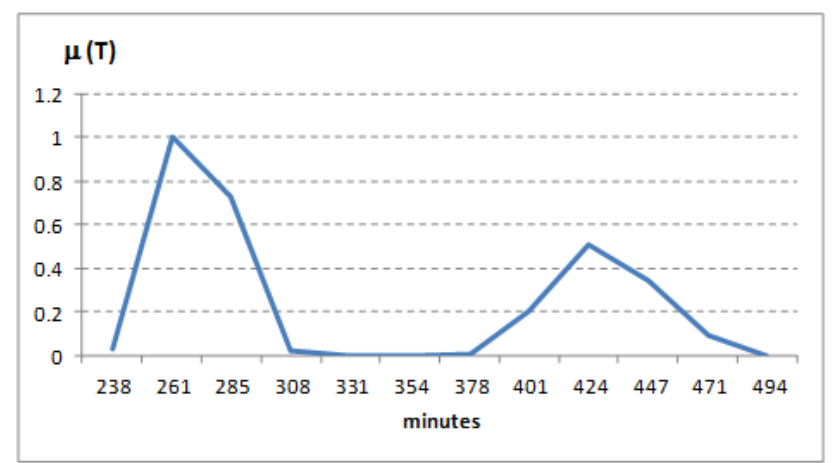

Figure 9. Possibility distribution of the intervention delay (normal conditions).

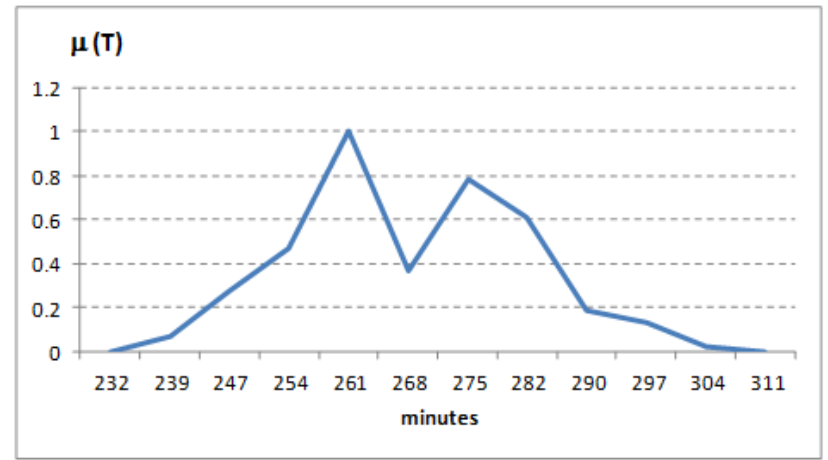

Figure 10. Possibility distribution of the intervention delay (bad visibility).

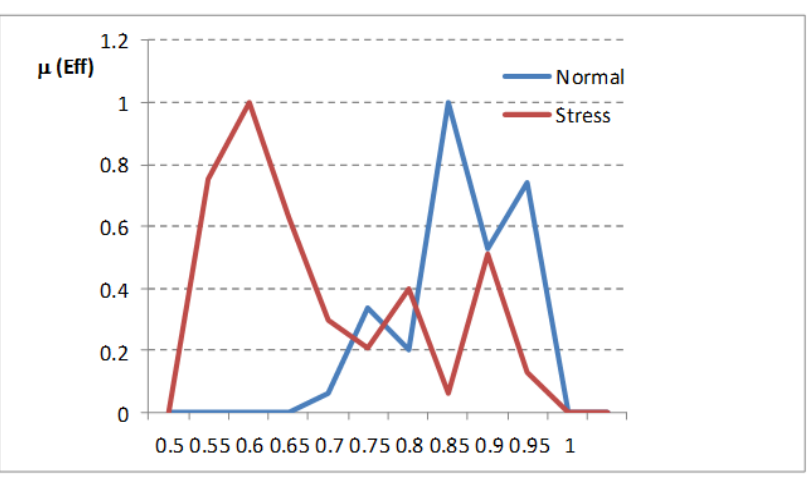

Figure 11. Procedure efficiency in normal and stress conditions.

\section{Discussion}

\subsection{Data}

The return of experience (REX) is essential to supply a knowledge base that could afterwards provide input data of a model (at least delay values since we focus on reactivity aspects), workable at each new instance. Even if events are unpredictable, deciders may have to face to situations that have previously occurred, which enforces the strong importance of REX in crisis management. Concerning an emergency system, these data are mainly:

a) The reaction delay of the emergency system consecutively to an urgent call.

b) The operational delay to treat all victims, function of their number, their seriousness, the experience of actors, etc. Human factors are not present in REX (especially after an exercise), where negative aspects are generally removed.

c) The delay of transport, under various conditions (weather, destination, hour, urban density, etc.). An emergency service covers is affected to a limited geographical area and because it is frequently requested, it is therefore possible to estimate increase coefficients to apply to data provided by a G. P. S. in a given situation.

The only limits of REX will concern crisis situations, after large scale accidents, where the formatted procedure has to take place to individual/ group initiatives. These situations may occur in downgraded conditions for logistics: no communication network, damaged roads.

\subsection{Model / Method}

Petri nets have been largely used to model operational processes and run simulations. They can also integrate fuzzy input-data. The emergency procedure is strongly formalized, that enables to translate it into a stable and generic model. The only-limitation lies in the representation of individual decisions. In this example, it has been represented (replaced) by their consequences: the delay to establish a report is representative of a stress and /or visibility conditions. The PN language is suitable to represent automated processes and their states. As the weight of human factors increases in a system (in crisis situations especially), it can be important to represent and insert qualitative notions concerning human behaviors, learning in action, dependencies, contagion (panic, fear, stress), optimization phenomena, internal compensation (an excess delay may be perceived by a group and then a collective reaction can lead to save time). As consequence in the operational model, it means that some parameters can be dynamically modified: thresholds, priority management, delays, etc. In a PN representation, corresponding parameters are: places markings, firing delays, weights of arcs, synchronization messages. To modify theses values dynamically, an upper level is required in the model, with logic operations to pilot these modifications, inference rules to take into account contextual variables. The global result is that the system will not only be reactive but it can become proactive, depending on statistical data, forecasting and/or learning methods.

\subsection{Interest of Simulation}

Simulation is for years a part of decision-making tools, in general situations. When an alert occurs, a simulation of the procedure model may be run, with (fuzzy) context parameters as input data. This can be used for resources provisioning and to estimate an intervention delay. It can also be pro-actively used to characterize intervention profiles, by combining different parameters of context. In that way, a simulation tool can be useful for learning, to run predefined scenarios and to test appropriate policies of resources 
management. For more general issues, it may be also combined with optimization tools in a hybrid approach (optimization and reactive simulation loops).

\section{Conclusion}

In this paper, a general method for modeling an emergency response system has been presented, taking into account uncertainties. Given the unpredictable nature of emergency, human factors are difficult to predict. The experience of previous situations if these situations are documented and indicated, should improve the decision support system. There are other sources of uncertainty, of more technical nature, but the method to represent them does not change. Possibility theory can represent any fuzzy number by using the data of experience and expertise. The advantage of fuzzy simulation is then to represent different assumptions, scenarios or configurations, to classify them according to a criterion of interest (we have used the response delay) and to manage resources accordingly.

\section{Acknowledgments}

This work was supported by the CPER project "AidCrisis" sponsored by the Champagne-Ardenne region and the French ministry of high education and research.

\section{References}

[1] J-L. Wybo and K. Madland-Kowalski, "Command centres and emergency management support", Safety Science, vol. 30, pp. 131-138, 1998.

[2] Z. Linz, "Organisationnal Performance under Critical Situation - Exploring the role of computer modelling in crisis case analysis", Computationnal and Mathematical Organization Theory, vol. 6, $\mathrm{n}^{\circ} 3$, pp. 277-310, 2000.

[3] C. Dautun, "Contribution to the study of hugh crisis, knowledge and decision-aided for civil security", $\mathrm{PhD}$ thesis, Sciences and environment engineering, Ecole des Mines de St-Etienne, France, 2007.

[4] C. Dautin, J. Tixier, F. Fontaine and G. Dusserre, "Crisis management: improvement of knowledge and developpement of a decision aid process", Loss Preventioon Bulletin, vol. 201, pp. 16-21, 2008.

[5] B. Balcik, "Relief chain planning and management: modelling and analyzing humanitarian logistic problem", Industrial engineering, Washighton University, 2000.

[6] C. Rongier, D. Gourc, M. Lauras and F. Galasso, "Toward a performance measurement system to control disaster responses", in Collaborative networks for a sustainable world, L. Camarinha-Matos and B. X. H Afsarmaneh, Eds. St-Etienne, France, 2010, pp. 189-196.

[7] C. Rongier, "Response to a crisis by performance management: towards a decision-aided tool, application to humanitarian", $\mathrm{PhD}$ Thesis, Industrial Engineering, INP Toulouse, France, 2012.
[8] M. Christopher, "Logistics and Supply Chain", Prentice-Hall, New York, 2005.

[9] R. Laroche, "Les événements naturels dommageables en France et dans le monde" (in French), Technical report, Ministry of Ecology, energy and sustainaible development, 2008.

[10] P. Lagadec, "La gestion de crises" (in French), Paris, Ediscience, 1991.

[11] L. Sayegh, W. Anthony and P. Perrewe, "Managerial decision-making under crisis: the role of emotion in an intuitive decision process", Human Resource Management Review, vol. 14, pp. 179-199, 2004.

[12] K. Kowalski-Trakofler, C. Vaught and T. Sharf, "Judgement and decision-making under stress, an overview of emergency managers", International Journal of Emergency Management, vol. $1, \mathrm{n}^{\circ} 3$, pp. 278-298, 2003.

[13] M. Seeger, "Chaos and crisis: proposition for a general theory of crisis communication", Public Relation Review, vol. 28, pp. 329-337, 2003.

[14] L. Weisaerth and O. Knudsen, "Technological disasters, crisis management and leadership stress", Journal of Hazardous Materials, vol. 93, n 1 , pp. 35-45, 2002.

[15] R. Billings, T. Milburn and M. Schaalman, "A model of crisis perception: a theoretical and empirical analysis", Administrative Science Quaterly, vol. 25, n², pp. 300-316, 1980.

[16] C. Cassandras and S. Lafortune, "Introduction to discrete-event systems", Springer US, New York, 2008.

[17] I. Dzelme-Berzina, "Mathematical logic and quantum finite state automata", Theoretical Computer Science, vol. 410, n²0, pp. 1952-1959, 2009

[18] M. Traore, E. Chatelet, E. Soulier and H. Gabbar, "Learning Diagnosis based on evolving fuzzy finite state automation", Fort-Worth TX, 2014 Sept. 29-Oct. 2, pp. 41-50.

[19] L. Zadeh, "Fuzzy sets", Information and Control, vol. 8, pp. 338-353, 1965.

[20] L. Zadeh, "Sets as a basis for a theory of possibility", Fuzzy Sets and Systems, vol. 1, pp. 308-335, 1978.

[21] D. Dubois and H. Prade, "Possibility Theory", Plenum Press, New York, 1988.

[22] D. Dubois and H. Prade, "The three semantics of fuzzy sets", Fuzzy Sets and Systems, vol. 90, pp. 141-150, 1997.

[23] L. Gomes and A. Steiger-Garçao, "Programmable controller design based on a synchronized-colored Petri net model and integrating fuzzy reasoning", Lecture Notes in Computer Science, vol. 935, pp. 218-237, Springer-Verlag, Berlin, 1995.

[24] J. Cardoso, R. Valette and D. Dubois, "Petri nets with uncertainty markings", Lecture Notes in Computer Science, vol. 483, pp. 64-78, Springer-Verlag, Berlin, 1991.

[25] S. Chanas and M. Nowakowski, "Single-value simulation of fuzzy variable", Fuzzy Sets and Systems, vol. 25, pp. 43-57, 1988. 
[26] D. Dubois and H. Prade, "Random sets and fuzzy intervals analysis", Fuzzy Sets and Systems, vol. 42, pp. 87-101, 1991.

[27] D. Gien and S. Jacqmart, Design and Simulation of manufacturing systems facing imperfectly defined information, Simulation Modeling Practice and Theory, vol. 13, n⿳6, pp. 465-485, 2005.
[28] P. Lallement, Simulation of IP networks, some guidelines based on A. I. and the fuzzy subseets theory, SICPRO, 2005, Jan. 25-28, Moscow, pp. 1016-1025.

[29] D. Dubois, H. T. Nguyen and H. Prade, "Possibility Theory, Probability and Fuzzy Sets", in Fundamentals of Fuzzy Sets, D. Dubois and H. Prade, Eds, Kluwer, Boston, 2000. 